\title{
Brownian particle in non-equilibrium plasma
}

\author{
B.I. Lev, V.B. Tymchyshyn, A.G. Zagorodny \\ Bogolyubov Institute for Theoretical Physics, NAS Ukraine, Metrologichna 14-b,03680, Kyiv, Ukraine
}

Received June 1, 2009

The stationary distribution function of Brownian particles in a nonequilibrium dusty plasma is calculated with regard to electron and ion absorption by grains. The distribution is shown to be considerably different from the distribution function of ordinary Brownian particles in thermal equilibrium. A criterion for the grain-structure formation in a nonequilibrium dusty plasma is derived.

Key words: Brownian motion, Langevine equation, Fokker-Planck equation, nonequilibrium

PACS: 05.70.Rr, 05.20.Dd, 05.40.-a

\section{Introduction}

The physics of dusty plasma attracts growing attention both as an academic problem and in view of technological applications; very recently it has become a subject of interest in the field of condense matter. A dust particle introduced in a plasma changes the properties of the latter. In astrophysics, dusty plasma has been found in planetary rings, in interplanetary and interstellar clouds as well as comets. Particles immersed in a laboratory plasma usually acquire negative charges since the diffusion flux of the electron component is higher than the ion flux. The parameters of a system of dusty particles can satisfy the condition for the formation of a Wigner or Coulomb crystal [1]. The dynamics of the formation and melting of such crystals was studied in [1] The particle dynamics during the structure formation process and the process of melting of a crystal structure should be described in terms of relevant physical conditions; it directly depends on the temperature and concentration of the relevant plasma component. A variety of physical processes can occur in a plasma, only sequential analysis can provide answers to many questions and thus enable us to understand the behavior of a system of particles immersed in a plasma. A very important issue is the accuracy of description of the dusty-plasma effective potential. The basic idea is to describe the absorption of electrons and ions by macroparticles in terms of effective point sinks appearing in the equation of plasma dynamics [1,3]. The approach makes it possible to find an explicit relation for the potential and the particle density distribution near the grains. The energy of grain interaction with the charge induced by the grain and the force acting on a moving dust particle depend on the particle velocity. In this case negative friction of the moving grains is possible. The standard approach to the description of the particle-velocity distribution function cannot be applied now and there arises the problem of how to find the average velocity of dust particles and to obtain a condition of crystal-structure formation in the grain subsystem. of macroparticles introduced in the plasma. In this paper we propose a self-consistent approach to the description of friction and diffusion of dust particles (grains) treated as Brownian particles[3]. The approach employs the nonlinear Fokker-Planck equation with regard to the velocity dependence of both friction and diffusion coefficients. We find the distribution function for dust particles in a plasma and derive conditions of a crystal structure formation in a system of dust macroparticles immersed in a weakly ionized plasma. We formulate a basic model for the general case of macro-particle Brownian motion in nonlinear media. Then we consider the macro-particle screening in a weakly ionized plasma and find the force that acts on each moving particle in such a system. With the force being known, we find the dust-particle distribution function and derive the structure-formation condition for this system. We conclude with the discussion of a possibility to observe the formation pattern experimentally. 


\section{Brownian motion of dusty particle in nonequilibrium plasma}

It is a matter of general knowledge that any statistical system interacting with the environment reaches its equilibrium state during the relaxation time. The properties of the combined system are determined by the physical peculiarities of the statistical system and the characteristics of the environment. Equilibrium state of a macroscopic system can be determined for some ideal conditions [4-7]. By virtue of the effect of the environment, the thermodynamic parameters of this system become equal to those for the thermal bath. In the case of an open system the combined parameters cannot be found. Such systems can, however, manifest equilibrium-like behavior. For example, in the case of a dusty plasma consisting of electrons, ions, and grains which absorb all the encountered electrons and ions, we can define the equilibrium state as a stationary state with equalized energy interchange between the grains and the environment. This state arises due to the balance between the direct energy transfer from the environment to the grain and the energy losses due to the processes that accompany the grain-motion relaxation. Experimental measurements of the grain temperature [8] show that the kinetic grain temperature can exceed the ion temperature by about two orders. This confirms that the behavior of the system can be indeed equilibrium-like. There are many other examples of such systems both in nature and in laboratories. These are hot electrons in semiconductors [6], photons scattered by an inhomogeneous medium with frequency-dependent diffraction coefficient [9], high-energy particles generated in the course of nuclear collisions in accelerators, ordinary Brownian particles in a medium with particle-velocity-dependent friction coefficient, etc. For such systems, characteristics of the media can be taken into account through the friction coefficient that depends on the phase-space point as a random quantity. Colloid particles in suspensions and grains in dusty plasmas provide good examples of systems with nonlinear particle-velocity dependencies of the kinetic coefficients. To describe the Brownian motion in a system with foreign particles is a key problem of the statistical physics of open systems. Stationary distributions of Brownian particles in a dusty plasma with a specified velocity dependence of the friction and diffusion coefficients were found in [10] for the case of the collisionless plasma background. The stationary distribution function has a maximum for some value of the velocity that determines the mean kinetic energy of the grains; the stationary one-particle distribution function is non-Maxwellian. The traditional description of the non-equilibrium Brownian motion employs phenomenological expressions for the friction and diffusion coefficients. Usually, the velocity dependencies of these coefficients are postulated, with a high level of uncertainty being thus introduced in the application of such models to real systems. Moreover, in the case of an open system there exist several types of Fokker-Planck equations which can be related to the nonlinear Langevine equation $[11,12]$. The dynamics of an ordinary Brownian particle is described in terms of velocity by the Langevin equation

$$
\dot{v}=-\gamma v+F(t)
$$

where $\gamma$ is the coefficient of friction, and $F(t)$ is a random force accounting for the irregular action of the environment on a separate particle. The average over the statistical ensemble $\langle F(t)\rangle=0$, but $\left\langle F(t) F\left(t^{\prime}\right)\right\rangle=\phi^{2} \delta\left(t-t^{\prime}\right)$, which corresponds to the white-noise condition and thus accounts for the correlations in the particle motion under the action of a random force. The random effect of the environment can be taken into account only through the correlations between fluctuations at various time instants $\left\langle L(t) L\left(t^{\prime}\right)\right\rangle=\phi\left(t-t^{\prime}\right)$. The mean value of the correlations $\phi \delta\left(t-t^{\prime}\right)$ is nonzero only during the correlation time. Therefore, the function must have a sharp peak as the time interval tends to zero, which corresponds to the condition that characterizes the white noise $[12,13]$. In the most general case we can use the Newton dynamic equation given by

$$
\dot{v}=f(v)+F(t)
$$

where $f(v) \equiv \frac{F(v)}{M}, M$ is mass of Brownian particle and $F(v)$ is the resulting force that acts on an individual particle including the contribution of the friction force. In the general case the resulting force in a nonlinear medium can depend on the particle velocity. This form of the dynamic equation for a Brownian particle can be regarded as nonlinear Langevine equation. The true nonlinear 
Langevine equation should be equivalent to the equation for the probability distribution function describing the physical process under consideration. Currently, there exist two different approaches to the solution of this problem. If one considers a Brownian particle for which the coefficient depends on the velocity at the starting point, then the equation for the non-equilibrium distribution function can be obtained in the Ito form. On the other hand, if this coefficient depends on the velocity before and after the transition, then the diffusion equation can be written in the Stratonovich form:

$$
\frac{\partial \rho}{\partial t}=-\frac{\partial}{\partial v} f(v) \rho+\frac{\sigma^{2}}{2} \frac{\partial}{\partial v} g(v) \frac{\partial}{\partial v} g(v) \rho
$$

In what follows only the Stratonovich presentation is used because both presentations are related $[12,13]$. All the solutions of these equations for the case of long evolution time possess a fundamental property, namely, they reduce to the stationary solution which can be regarded as an "equilibrium" solution for this system. Such stationary solution complies with the H-theorem in its general meaning [12]. The processes under consideration require physical interpretation. The equation for the non-equilibrium distribution function can thus be rewritten in a more usual form of the local probability conservation law, i. e.,

$$
\frac{\partial \rho(v, t)}{\partial t}=\frac{\partial J(\rho(v, t))}{\partial v} .
$$

In turn, the probability flow can be written as:

$$
J=-\left(f(v)-\frac{\sigma^{2}}{2} g(v) \frac{\partial}{\partial v} g(v)\right) \rho+\frac{\sigma^{2}}{2} g^{2}(v) \frac{\partial}{\partial v} \rho .
$$

The stationary solution of the Fokker-Planck equation for $J(\rho(v, t))=0$ is given by

$$
\rho_{s}(v)=A \exp \left\{\int \frac{2 f\left(v^{\prime}\right) \mathrm{d} v^{\prime}}{\sigma^{2} g^{2}\left(v^{\prime}\right)}-\ln g(v)\right\} .
$$

The equilibrium distribution function as a stationary solution in the general non-equilibrium case is given by

$$
\rho_{s}(v)=A \exp \{-U(v)\}
$$

where

$$
U(v)=\ln g(v)-\int \frac{2 f\left(v^{\prime}\right)}{g^{2}(v)} \mathrm{d} v^{\prime} .
$$

This distribution function has an extreme value, which can be found as a solution of the equation

$$
U^{\prime}(\tilde{v})=\frac{1}{D(v)}\left(D^{\prime}(v)-f(v)\right)
$$

where symbol ' denotes the energy derivative. This equation is equivalent to the one

$$
D^{\prime}(\tilde{v})=f(\tilde{v})
$$

which gives a relation between the dissipation and diffusion in the stationary case and completely determines the new "equilibrium" state of the system. The stationary non-equilibrium distribution function is given by

$$
\rho_{s}(v)=\exp \{-U(\tilde{v})\} \exp \left(-U^{\prime \prime}(\tilde{v}) v^{2}\right)
$$

where

$$
U^{\prime \prime}(\tilde{v})=-\frac{1}{D(\tilde{v})}\left(D^{\prime \prime}(\tilde{v})+f^{\prime}(\tilde{v})\right)
$$

This "equilibrium" distribution function is Gaussian in all the cases. When the system dissipation $f(v)$ is described by the nonlinear function of state and the diffusion coefficient depends on 
the velocity, many interesting cases, including the noise-induced transition in a more stable new "equilibrium" state, can be considered. The velocity dependence of the diffusion coefficient can be also derived from the linear Langevin equation in terms of the theory of Markovian processes with regard to the non-equilibrium fluctuations of various coefficients which determine the function. This approach can also be used to consider a very simple picture of the Brownian motion in heterogeneous media. For this case the characteristics of the medium can be taken into consideration by means of a varying friction coefficient which depends on the space point as a random quantity. This means that we have to put in the Langevine equation, i. e., the friction coefficient consists of the constant part which determines the average friction coefficient, and the random part which describes the effect of random variations of the friction properties of the matter. In the case of white-noise spectrum of density fluctuations or other parameters which characterize the matter, the Fokker-Planck equation can be written as [13]:

$$
\frac{\partial \rho(v, t)}{\partial t}=\frac{\partial}{\partial v}(\gamma v \rho(v, t))+\frac{\sigma^{2}}{2} \frac{\partial^{2}}{\partial v^{2}} v^{2} \rho(v, t)
$$

The stationary solution of this equation is given by [13]:

$$
\rho_{s}(\varepsilon, t)=N v^{-\left(\frac{2 \gamma}{\sigma^{2}}+1\right)}
$$

which can be verified by direct substitution into equation (13). This stationary solution considerably differs from that for the case of velocity-independent kinetic coefficients. Let us assume that there exists a mechanism responsible for the velocity limitation. For example, for the dissipation function $f(\varepsilon)=\gamma \varepsilon-\varepsilon^{2}$, such limitation follows from the second part. The absorption parameter can be presented as $\gamma_{t}=\gamma+\xi_{t}$ where the second part describes the random change of the environmental effect. In this case the Fokker-Planck equation has the form [13]:

$$
\frac{\partial \rho(v, t)}{\partial t}=\frac{\partial}{\partial v}\left(\left(\gamma v^{2}\right) \rho(v, t)\right)+\frac{\sigma^{2}}{2} \frac{\partial^{2}}{\partial v^{2}} v^{2} \rho(v, t) .
$$

The stationary solution of this equation is given by

$$
\rho_{s}(v, t)=N v^{-\left(\frac{2 \gamma}{\sigma^{2}}+1\right)} \exp \left\{-\frac{2}{\sigma^{2}} v\right\} .
$$

The latter statement can be verified by a direct substitution into equation (13). This stationary solution considerably differs from that for the case of velocity-independent kinetic coefficients. For Rayleigh's phenomenological model of active friction $\gamma_{t}=\gamma-\alpha v^{2} \equiv \alpha\left(v^{2}-v_{0}^{2}\right)$, where $v_{0}^{2}=\frac{g}{\alpha}$ with the constant diffusion coefficient $D(v) \approx D_{0}$, the stationary velocity distribution function is given by

$$
\rho_{s}(v, t)=N \exp \left\{-\frac{\alpha}{4 D_{0}}\left(v^{2}-v_{0}^{2}\right)^{2}\right\},
$$

where $N$ is a normalization constant. In the weak noise limiting case $\frac{\alpha}{4 D_{0}} \rightarrow 0$, this distribution is transformed into $\delta$-the function and thus the dispersion of the kinetic energy is completely neglected. The Brownian particle then moves with constant kinetic energy $\frac{m_{g} v_{0}^{2}}{2}$ while its velocity is randomly changed with time. For this simple model, we can find the condition for the formation of a spatial periodic structure with regard to the potential interaction energy. In the next section we consider a more realistic model of grain behavior in a dusty plasma.

\section{Macroparticle screening in weakly ionized plasma}

The purpose of this section is to find the force acting on an individual particle in a weakly ionized plasma. The appropriate calculations for the case of collisionless plasma were obtained in $[14,15]$ on the basis of the microscopic treatment. In the case of the grain moving in weakly-ionized 
plasma the basic set of equations for individual component concentration variations can be written in the form $[1,2]$

$$
\left.\left.\frac{\partial n_{\alpha}(r, t)}{\partial t}-\operatorname{div} \Gamma_{\alpha}=I_{0}-\beta n_{e} r, t\right) n_{i} r, t\right)-S_{\alpha} \delta(\mathbf{r}-\mathbf{v} t)
$$

and the Poisson equation for the potential is given by

$$
\Delta \Phi(r, t)=-4 \pi \sum_{\alpha} e_{\alpha} n_{\alpha}(r, t)-4 \pi q \delta(\mathbf{r}-\mathbf{v} t)
$$

where $v$ is velocity of grain, $q$ and $S$ can be treated as constant and determined concentrated charge and intensity of the point sink. As far as its form is rather involved, we shall not reproduce it here. Instead we give an expression for the force that should be considered in order to describe the dynamics of dust particles in the plasma. The force acting on the grain due to the existence of an induced potential is described by the relation

$$
\Phi(r, t)=(q+S) \frac{\exp \left(-k_{\mathrm{D}}\right) r}{r}-\frac{S}{r},
$$

where $k_{\mathrm{D}}$ is inverse screening length. For the moving grains, electrostatic potential has been obtained in article [20] and it has got a very cumbersome form, which we are not going to cite here, but we do cite the force which must be given consideration to describe the dynamic dusty particles in plasma. In fact, the force acting on the grain due to the existence of the induced potential is described by the relation $F(v, \alpha)=-q \lim _{r \rightarrow v t} \frac{\partial \Phi(r, t)}{\partial r}$. The force produced by the particle-cloud interaction is given by [20]:

$$
\begin{aligned}
F(\tilde{v}, \alpha)= & \frac{\mathrm{i} q^{2} k_{\mathrm{D}}^{2}}{\pi} \int_{0}^{\infty} \frac{\mathrm{d} x}{\tilde{v}^{2}\left(\mu_{1}-\mu_{2}\right) d}\left\{2 \mathrm{i} \tilde{v} \frac{t+d}{t+1}\left(\mu_{1} \mu_{2}\right)+\mu_{1}\left[\mathrm{i} \mu_{1} \tilde{v} \frac{t+d}{t+1}-x(1-\alpha)\right] \ln \frac{\mu_{1}-1}{\mu_{1}+1}\right. \\
& \left.-\mu_{2}\left[\mathrm{i} \mu_{2} \tilde{v} \frac{t+d}{t+1}-x(1-\alpha)\right] \ln \frac{\mu_{2}-1}{\mu_{2}+1}\right\} .
\end{aligned}
$$

We introduce a coefficient

$$
\mu_{1,2}=\frac{\mathrm{i}\left[x^{2}(1+d)+\frac{t+d}{t+1}\right]}{-2 x \tilde{v} d} \pm \frac{\sqrt{4 x^{2} d\left(1+x^{2}\right)-\left[x^{2}(1+d)+\frac{t+d}{t+1}\right]^{2}}}{-2 x \tilde{v} d}
$$

where $\tilde{v}=\frac{v}{D_{\mathrm{i}} k_{\mathrm{D}}}, d=\frac{D_{\mathrm{i}}}{D_{\mathrm{e}}}, t=\frac{T_{\mathrm{i}}}{T_{\mathrm{e}}}, \alpha=-\frac{\tilde{S}}{q}$, here $D_{\mathrm{i}}$ and $D_{\mathrm{e}}$ are ion and electron diffusion coefficient, $T_{\mathrm{i}}$ and $T_{\mathrm{e}}$ - temperature of ion and electron plasma components respectively. $q$ - charge of particle that can be calculated as:

$$
q=-\frac{T_{\mathrm{e}} r_{0}}{e} \ln \left(\frac{\mu_{\mathrm{e}}}{\mu_{\mathrm{i}}}\right)
$$

$\mu_{\mathrm{i}}$ and $\mu_{\mathrm{e}}$ are ion and electron mobilities respectively, $e$ is electron charge. The model of a point sink makes it possible to obtain an analytic solution of the problem of grain screening and to find explicitly the asymptotical behavior of the potential and the force which acts on several grains in a weakly ionized plasma. After this we can explain the dynamics of the grain system and estimate the probable conditions for crystal structure formation in such systems. To this end, we can obtain the distribution function for the grains regarded as Brownian particles in non-equilibrium media.

\section{Stationary distribution function of grains in nonequilibrium plasma}

As follows from the previous section, the description of an aggregate dust particle can use the general form of the kinetic equation for particle distributions in various media. First of all we use the standard Fokker-Planck equation with velocity-independent diffusion coefficient. This equation can be written in the form:

$$
\frac{\partial \rho}{\partial t}=-\frac{\partial}{\partial v}(\gamma \rho)+\frac{\partial^{2}}{\partial v^{2}} D \rho .
$$


The solution of the latter is

$$
\rho(v, \alpha)=\frac{C}{D(v, \alpha)} \exp \left\{-\int \frac{f(v, \alpha) v}{D(v, \alpha)} \mathrm{d} v\right\}
$$

its value is maximum for the velocity that depends on the coupling parameter. This velocity can be treated as the equilibrium velocity of a Brownian grain in the plasma. Obviously, this velocity differs from the conventional thermal velocity. Its value is determined by the energy balance between the energy obtained by the grain due to ion absorption from the plasma, and the energy dissipation associated with the friction. Notice that large velocity cannot be attained since the distribution function has a maximum at some velocity. In order to get some analytical estimates let us consider $[16,17]$ of the order of one. In such a case the stationary solution for the distribution function possesses a maximum for non-zero velocity. Figure 1 shows the result of computer calculations of a stationary distribution function for the case of constant diffusion coefficients $D_{\mathrm{i}}=0.0542 \frac{\mathrm{cm}^{2}}{\mathrm{sec}}$, $D_{\mathrm{e}}=2580 \frac{\mathrm{cm}^{2}}{\mathrm{sec}}, D=0.016 \frac{\mathrm{cm}^{2}}{\mathrm{sec}}$ and $\eta=2,27 \times 10^{-4} \frac{\mathrm{cm}}{\mathrm{cm} \mathrm{sec}}$ As follows from the picture, the stationary distribution function has a sharp peak for nonzero grain velocity that depends on plasma temperature. Another important result is the asymmetric smearing of the distribution function that depends on possible fluctuations of the coupling parameter. The dependence of the probability distribution function on the grain velocity and temperature is given in figure 2 . Here and in what follows for the sake of simplicity we consider the case of isothermal plasma $T=T_{i}=T_{e}$.

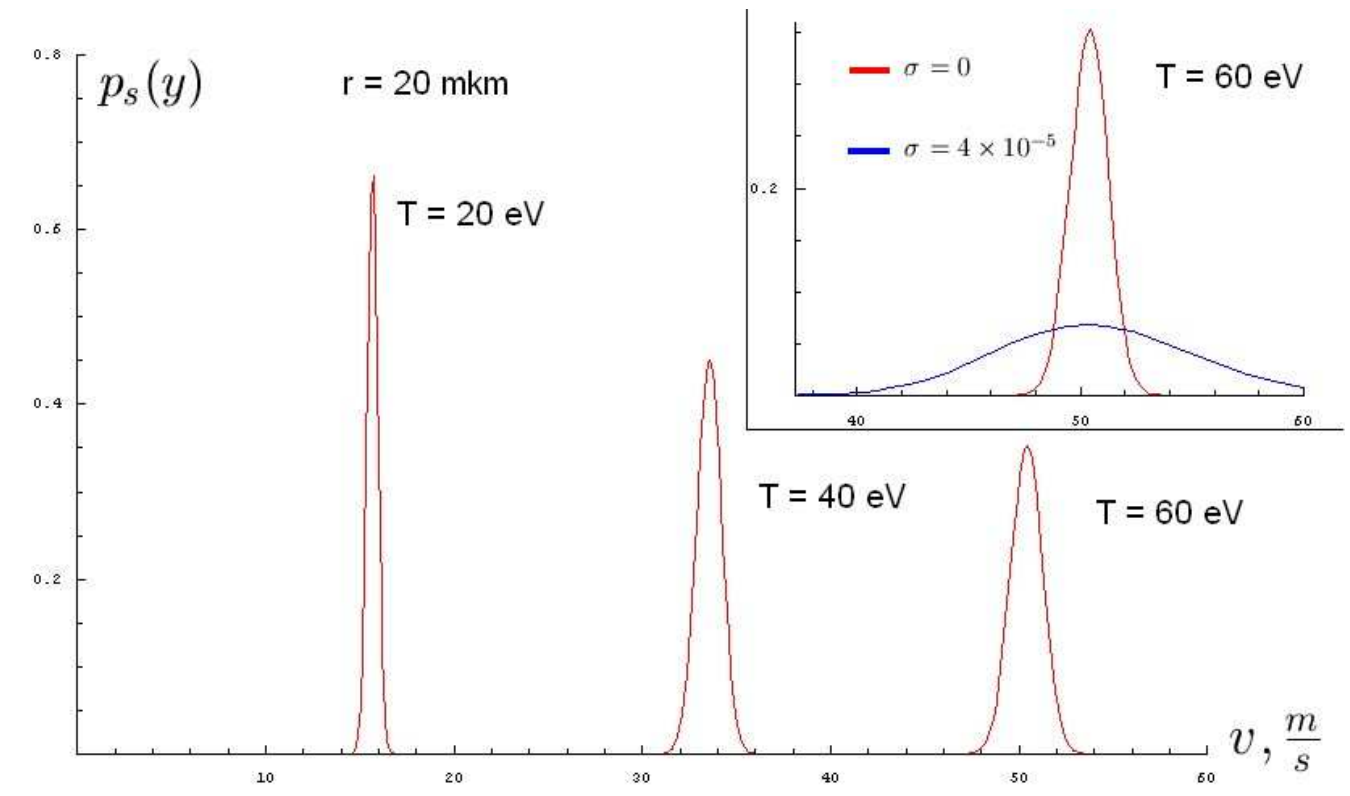

Figure 1. Probability distribution function for constant diffusion coefficients for different temperatures $\left(T=T_{\mathrm{e}}=T_{\mathrm{i}}\right)$ when $\alpha$ is stable. The plot in the upper right corner shows the difference between the probability distribution function for stable $\alpha$ and for the case when $\alpha$ fluctuates with dispersed $\sigma$ density.

The dependence of the distribution function on the grain velocity and radius for fixed temperature is shown in figure 3 . The dependence of the velocity corresponding to the local maximum of the distribution function on the grain radius and temperature is given in figures 4 and 5 .

Figures 6 and 7 present the dependence of the grain distribution function on the grain velocity and plasma temperature. The numerical calculations show that the qualitative changes of the grain distribution function can occur with the variation of both plasma and grain subsystem parameters If the distribution function has a local maximum for zero velocity, then the condition of Wigner crystal structure formation is completely determined by concentration of grains. In this case there exists only repulsive electrostatic interaction and mean concentration can determine 


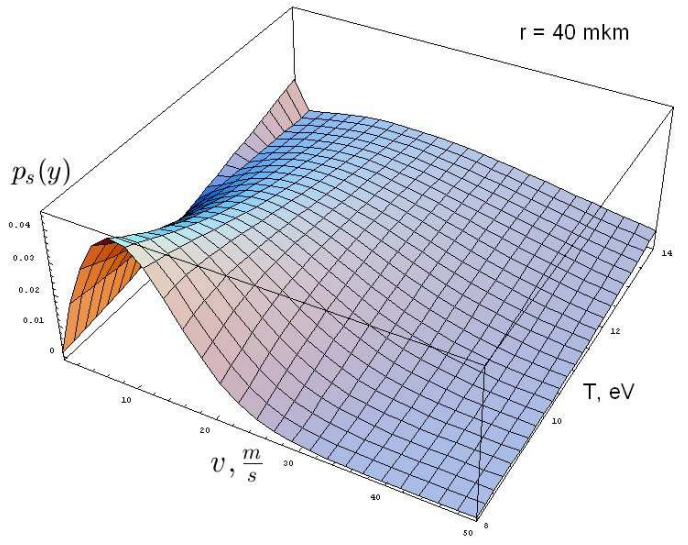

Figure 2. The dependence of the probability distribution function on the grain velocity and temperature $\left(T=T_{\mathrm{e}}=T_{\mathrm{i}}\right)$. Grain radius is fixed $(40 \mathrm{mkm})$.

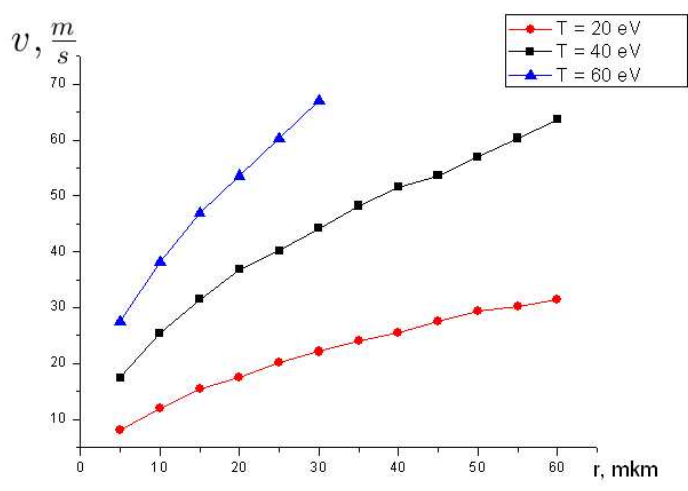

Figure 4. Grain-radius dependence of the grain velocity in the local maximum of the probability distribution function. The temperature is fixed $\left(T=T_{\mathrm{e}}=T_{\mathrm{i}}\right)$.

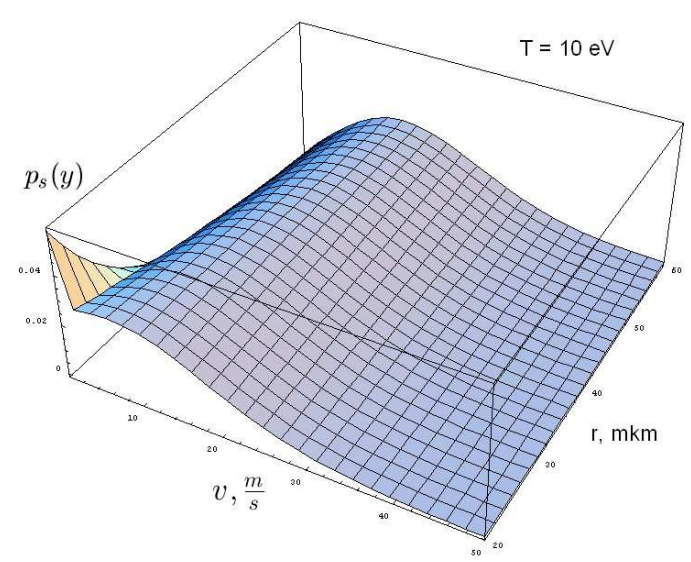

Figure 3. The dependence of the probability distribution function on the grain velocity and radius. Temperature $\left(T=T_{\mathrm{e}}=T_{\mathrm{i}}\right)$ is fi$\operatorname{xed}(10 \mathrm{eV})$.

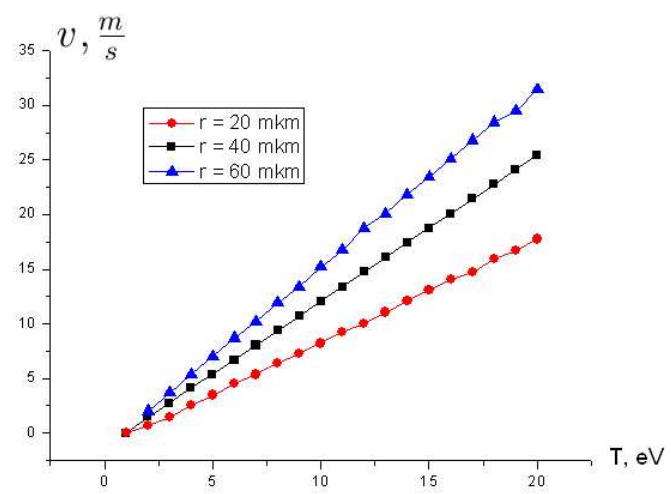

Figure 5. Temperature dependence of the grain velocity of in the local maximum of the probability distribution function.

spatially homogeneous distribution of grains. If the mean kinetic energy is nonzero, as in our case, and is equal to the potential interaction energy, one may expect a spatially ordered grain structure to be formed in the dusty plasma (Wigner-crystal-type structure). An essential element in any self-organization of condensation in a many-particle system is the condition that the potential due to the nearest- neighbor force must be greater than the thermal energy. In our case the criterion is satisfied if the average value of the velocity is zero. In more realistic cases, however, the average velocity is determined by the maximum distribution function which depends on the grain radii and plasma temperature; In the present case we can find the critical parameter for the formation of such structures in a weakly ionized dusty plasma. We find the kinetic energy of the dusty plasma and compare it to the interaction energy for an average distance. Thus we obtain a relation given by

$$
\left(\frac{m_{g}}{m_{i}}\right)^{\frac{1}{2}} \simeq \frac{Z_{g} R}{Z_{i} \lambda_{\mathrm{D}}} .
$$

The latter may be used to find the parameter of the periodic distribution of grains in the plasma. This condition determines the parameters of the plasma and grains for which a periodic grain 
structure can be formed in the plasma. For a real parameter of grains $R \sim 10 \mu m-200 \mu m$, the Debye lengths $\lambda_{\mathrm{D}} \sim 1000 \mu \mathrm{m}$, and the relation between the masses being given by $M \sim 10^{4} \mathrm{~m}_{i}$, the periodic structure can be realized for $Z_{g} \sim 2 \cdot 10^{4} Z_{i}$ which is a realistic parameter for the grains in a dusty plasma.

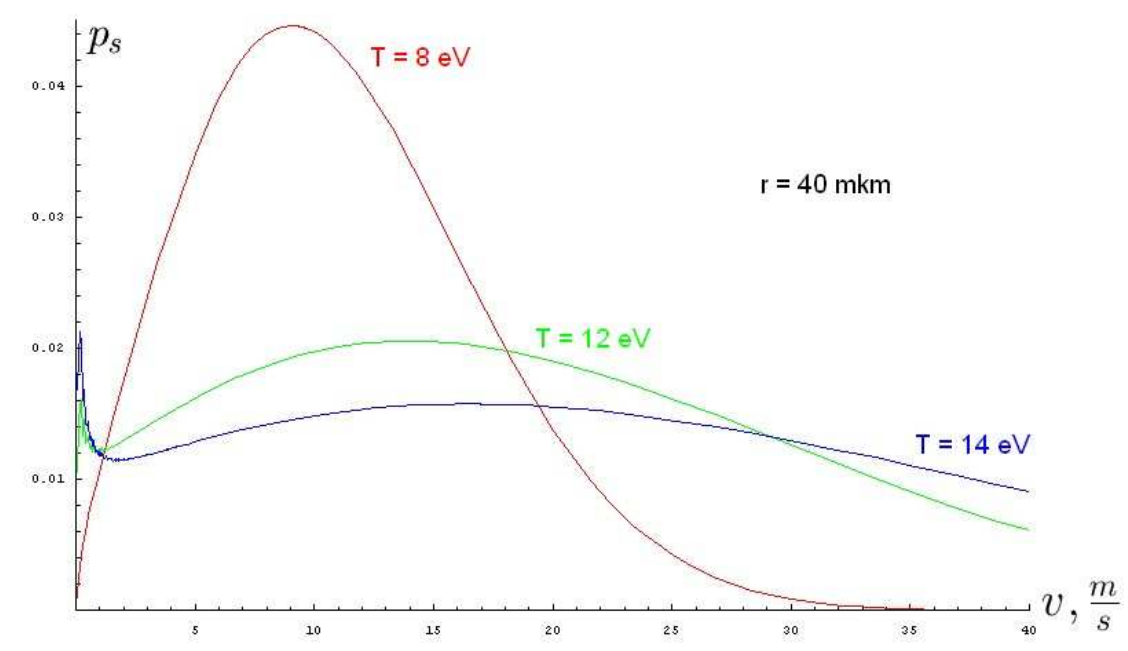

Figure 6. Grain-velocity dependence of the probability distribution function for different plasma temperatures $\left(T=T_{\mathrm{e}}=T_{\mathrm{i}}\right)$. The radius is fixed $(40 \mathrm{mkm})$. As temperature increases, the probability distribution function acquires two local maxima. For some values of the parameters, one may expect a bifurcation to appear.

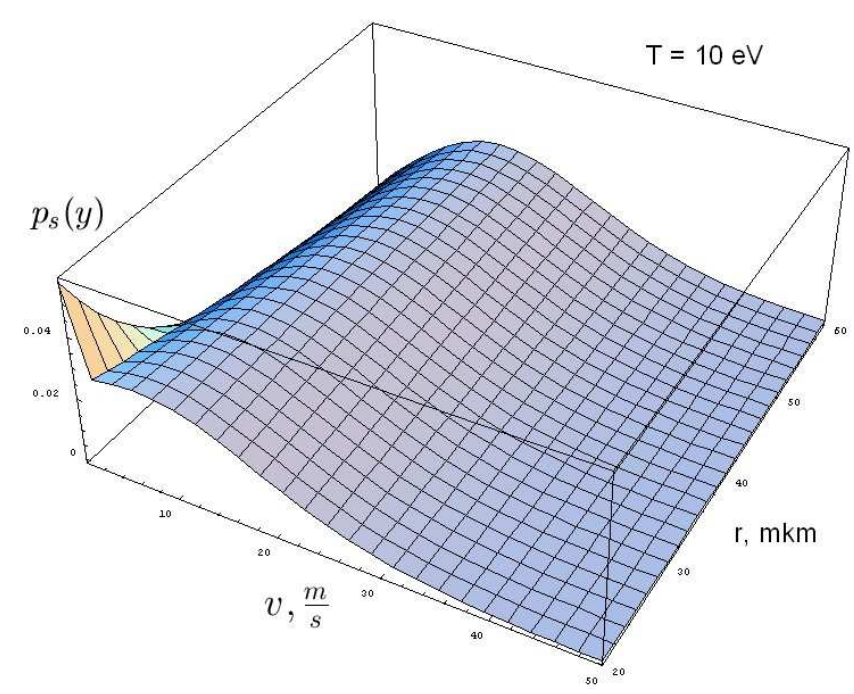

Figure 7. Grain-velocity dependence of the probability distribution for different grain radii. The temperature $\left(T=T_{\mathrm{e}}=T_{\mathrm{i}}\right)$ is fixed $(10 \mathrm{eV})$. As the radius decreases, the probability distribution function can acquire two local maxima. For some values of the parameters, one may expect a bifurcation to appear.

It should be noted that the latter estimates can also be used in the case of a fully-ionized plasma since the kinetic coefficients were derived disregarding electron and ion collisions with neutrals. Obviously, such collisions are extremely important in the case of a weakly ionized plasma. Nevertheless, the idea to calculate the average kinetic energy from the Fokker-Planck equation with velocity-dependent kinetic coefficients and then to compare this energy to the interaction energy still remains valid in the case of weakly ionized plasmas. In this case the main contribution to the 
diffusion is associated with the grain interaction with neutrals and thus the diffusion coefficient can be approximated by the appropriate expression for neutral grains in a neutral gas. As for the friction coefficient, it considerably depends on the velocity [16-19]. Moreover, under certain conditions [16-19] it can take negative values, as in the case of a fully-ionized plasma. Thus, the conditions for the formation of a dusty crystal in a weakly ionized plasma can be also estimated.

\section{Conclusion}

To conclude we notice that a new approach to the problem of grain structure formation in dusty plasmas is proposed. The stationary non-equilibrium solution of the Fokker-Planck equation for the Brownian motion of grains in a plasma is obtained. This solution can be interpreted as an "equilibrium" distribution function for a new steady state in the open system. This state is characterized by the "equilibrium" velocity of foreign particles. The approach takes into account probable transitions between different states of the system induced by the energy dissipation and the interaction with the environment dependent on the velocity. For a dusty plasma, as for an open system, the fluctuation-dissipation theorem in the Einstein-relation form is inapplicable. Therefore, microscopic approaches to this problem in terms of specific models are important and useful. Nonlinear models describing stationary states of the system are proposed and conditions of periodic structure formation in the grain subsystem are obtained. The presented numerical results make it possible to find the condition for the formation of a spatial periodic structure in the grain system. This research is partially supported by the joint NASU-RFFR grant.

\section{References}

1. Melzer A., Homann A., Piel A., Phys.Rev. E, 1996, 53, 2757.

2. Filippov A.V., Zagorodny A.G., Pal' A.F., Starostin A.N., Momot A.I., JETP, 2009, 135, 567.

3. Lev B.I., Zagorodny A.G., Phys. Lett. A, 2009, 373, 158.

4. Balesku R. Equilibrium and Nonequilibrium Statistical Mechanics. J. Wiley and Sons, New York, 1978.

5. Landau L.D. Statistical Mechanics. Nauka, Moskow, 1973.

6. Zubaryev D. Nonequilibrium Statistical Thermodynamics. Nauka, Moskow, 1971.

7. Huang K. Statistical Mechanics. J. Wiley and Sons, New York, 1963.

8. Morfill G.E., Thomas H.M., Konopka U., Zuzic M., Phys. Plasma, 1999, 6, 1769.

9. Gardiner C.W., Zoller P. Quantum Noise. Springer, 2000; Wells D.F., Milburn G.J. Quantum Optics. New Zeland, 2001.

10. Trigger S.A., Zagorodny A.G., Cond. Matter Phys., 2004, 7, 629.

11. Klimontovich Yu.L. Theory of Open System. Kluwer A. Publication, Dordrecht, 1995.

12. van Kampen N.G. Stochastic Process in Physics and Chemistry. North-Holland, Amsterdam, 1990.

13. Horsthemke W., Lefever R. Noise-induced transition (Theory, applications in physics, chemistry and biology). Springer-Verlag, New-York, 1984.

14. Zagorodny A.G., Schram P.P.J.M., Trigger S.A., Phys. Rev. Lett., 2000, 84, 3594.

15. Schram P.P.J.M., Sitenko A.G., Trigger S.A., Zagorodny A.G., Phys. Rev. E, 2000, 63, 016403.

16. Zagorodny A.G., Filippov A.V., Pal' A.F., Starostin A.N., Momot A.I. J. Phys. Stud., 2007, $11,158$.

17. Khrapak S.V., Zhdanov S.A., Ivlev A.V., Morfill G.E., J. Appl. Phys., 2007, 101, 033307.

18. Vladimirov S.V., Khrapak S.A., Chaudhuri M., Morfill G.E., Phys. Rev. Lett., 2008, 100, 055002.

19. Filippov A.V., Zagorodny A.G., Pal' A.F., Starostin A.N., Momot A.I., JETP Letters, 2008, 88, 24. 


\title{
Броунівська частинка у нерівноважній плазмі
}

\author{
Б.І. Лев, В.Б. Тимчишин, А.Г. Загородній
}

Інститут теоретичної фізики ім. Боголюбова НАН України, Метрологічна 14-б,03680, Київ, Україна

Отримано 1 червня 2009 р.

Знайдена стаціонарна функція розподілу для Броунівських частинок, що знаходяться в в нерівноважній запорошеній плазмі. Для цього використано нелінійне рівняння Фоккера-Планка. Сила, що діє на частинку, враховує безпосередній вплив плазми за рахунок зміни поля рухомої частинки при абсорбції зарядів на її поверхні та звичайне тертя порошинки в плазмі. Показано, що стаціонарна функція розподілу по швидкостях для частинок не відповідає рівноважному розподілу для звичайних Броунівських частинок. Знайдені критерії формування структур в системі таких частинок в нерівноважній плазмі.

Ключові слова: Броунівський рух, рівняння Ланжевена, рівняння Фоккера-Планка

PACS: 05.70.Rr, 05.20.Dd, 05.40.-a 